\title{
Foreword
}

\section{Cardiac Implantable Electronic Devices}

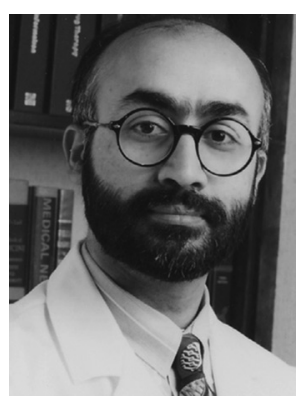

Ranjan K. Thakur, MD, MPH, MBA, FHRS

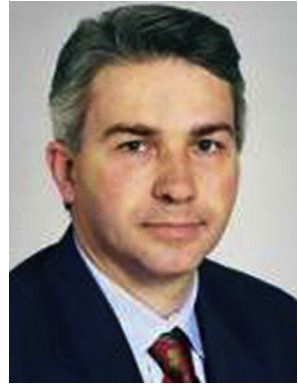

Andrea Natale, MD, FACC, FHRS

Consulting Editors

Care of patients with cardiac implantable electronic devices (CIED) comprises the bulk of time most cardiac electrophysiologists in clinical practice spend providing patient care. Thus it's appropriate to discuss current thinking on various issues related to management of patients with CIEDs. We are pleased that Drs Al-Ahmad, Yee, and Link have assembled a respected panel of contributors and put together this excellent issue of Cardiac Electrophysiology Clinics on contemporary review of CIED with future directions.

This volume is clinically oriented and covers many contemporary topics of interest to clinicians, such as the use of wearable defibrillators during the waiting phase before an implantable defibrillator is implanted, current status and future developments in leadless pacemakers, His bundle pacing, use of single- or dual-coil defibrillator leads, inappropriate implantable cardioverter defibrillator shocks, device-detected atrial fibrillation, procedural issues, technology issues, and controversies about contemporary practice issues.
This volume will be of interest to all clinical electrophysiologists as well as cardiology and electrophysiology fellows in training and lab and nursing personnel who help us take care of these complex patients. This volume is easy to read because it deals with subjects that are familiar to all of us, well presented, and clearly written. We hope you enjoy it.

Ranjan K. Thakur, MD, MPH, MBA, FHRS Sparrow Thoracic and Cardiovascular Institute 1400 East Michigan Avenue Suite 400

Lansing, MI 48912, USA

Andrea Natale, MD, FACC, FHRS Texas Cardiac Arrhythmia Institute

Center for Atrial Fibrillation at

St. David's Medical Center 1015 East 32nd Street, Suite 516 Austin, TX 78705, USA

E-mail addresses:

thakur@msu.edu (R.K. Thakur) andrea.natale@stdavids.com (A. Natale) 